\title{
Pocket-creation method using a new technique of single-clip traction for colorectal endoscopic submucosal dissection
}

Colorectal endoscopic submucosal dissection (ESD) is a challenging procedure used in the removal of colorectal neoplasms and various methods have been developed to improve its performance [1-3]. Among these, the pocket-creation method (PCM) allows the safe and efficient removal of neoplastic lesions, using precise submucosal dissection just above the muscularis propria [4, 5]. However, the pocket-opening phase of PCM is often cumbersome and time-consuming. We have invented a new technique using single-clip traction that we have called PCM with clip traction (PCM-CT), which we believe improves the speed and safety of the pocket-opening phase. PCM-CT creates submucosal traction by using a single hemoclip to affix normal mucosa from the distal side of the lesion to normal mucosa on the opposite colonic wall ( $\triangleright$ Fig.1). This elongates the submucosa, enabling clearer identification of the safest submucosal dissection line.
A 20-mm, Paris lla, nongranular, JNET 2B, laterally spreading lesion in the proximal ascending colon (diagnosed on pathology as being a well differentiated slightly invasive submucosal adenocarcinoma with a 400- $\mu \mathrm{m}$ invasion depth, negative lymphovascular invasion, and negative margins) was removed using the PCM-CT ( Fig. 2; Video 1). As per the standard PCM, a submucosal pocket was first created below the lesion. Next, a circumferential mucosal incision was performed approximately $7 \mathrm{~mm}$ outside the margin
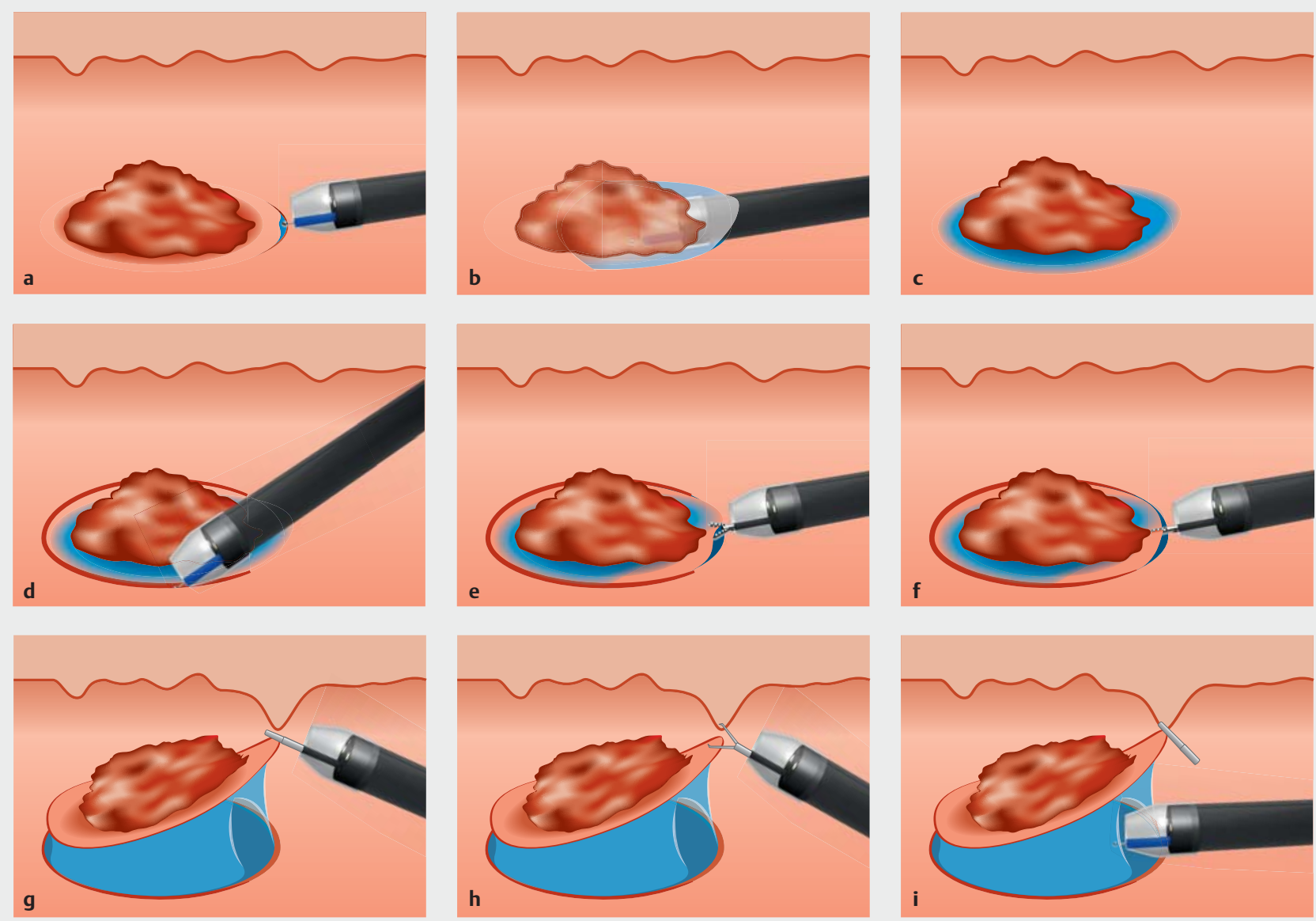

Fig. 1 Illustration of the pocket-creation method with clip traction (PCM-CT). a - c The submucosa below the lesion is dissected using the PCM; $\mathbf{d}$ a circumferential mucosal incision is created around the lesion; $\mathbf{e}, \mathbf{f}$ a reopenable hemoclip is used to grasp normal mucosa on the distal edge of the partially resected lesion, without deployment; $\mathbf{g}$ the entrapped mucosa is pulled towards the normal mucosa on the opposite intestinal wall; $\mathbf{h}$ the hemoclip is slowly reopened to capture the opposing normal mucosa and the clip is deployed; $\mathbf{i}$ the remaining submucosa below the lesion is dissected with reliable identification of the safest submucosal dissection line. 

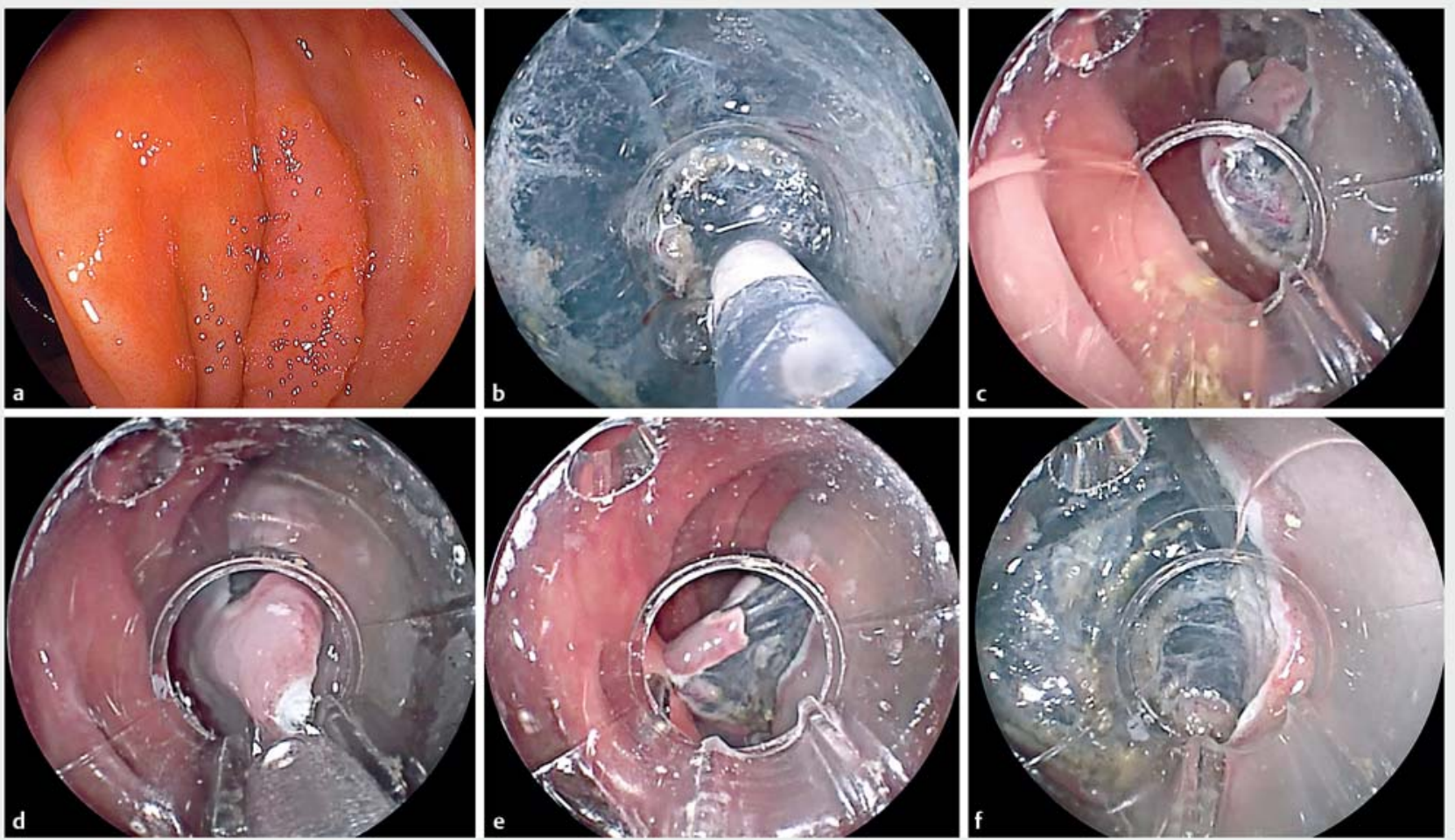

Fig. 2 Endoscopic images showing: a a 20-mm, Paris lla, nongranular, JNET 2B, laterally spreading lesion in the ascending colon; b colonic endoscopic submucosal dissection (ESD) being performed using the pocket-creation method (PCM); $\mathbf{c}$ a circumferential incision being made in the normal mucosa around the lesion after the submucosal pocket has been created; $\mathbf{d}$ normal mucosa on the distal edge of the lesion being captured with a reopenable clip; e the clip, with the captured mucosa from the edge of the lesion, being pulled towards the opposite colonic wall and reopened to entrap the opposing normal colonic mucosa as well; $\mathbf{f}$ the identification of the safest submucosal dissection line, which is improved after PCM with clip traction.
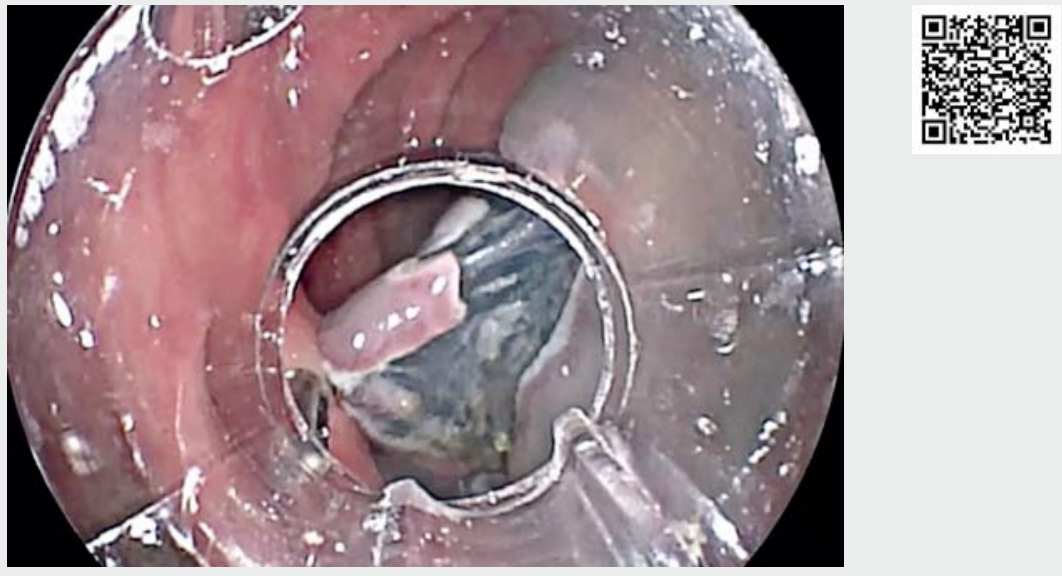

Video 1 The pocket-creation method with clip traction (PCM-CT) is performed on a 20-mm laterally spreading lesion in the proximal ascending colon.

of the lesion. A reopenable hemoclip (SureClip; Micro-Tech Co. Ltd., Nanjing, China) was used to grasp normal mucosa on the distal edge of the partially resected lesion, without being deployed. The resulting entrapped mucosa was then pulled towards the opposite colonic wall, where the hemoclip was carefully reopened to capture the opposing normal colonic mucosa. Once it was confirmed that both mucosal sites had been secured, the hemoclip was deployed. The remaining submucosa beneath the lesion was then dissected and the lesion was safely removed.

In conclusion, PCM-CT provides continuous submucosal traction on lesions by stretching the submucosa and allowing clearer identification of the safest submucosal resection path. This technique facilitates the pocket-opening stage of PCM and potentially improves its safety and efficiency.

Endoscopy_UCTN_Code_TTT_1AQ_2AD

Competing interests

H. Yamamoto has a patent for ESD devices produced by the Fujifilm Corporation. He also has a consultant relationship with the Fujifilm Corporation and has received honoraria, grants, and royalties from the company. 
The authors

Tatsuma Nomura ${ }^{1}$, Yoshikazu Hayashi ${ }^{1}$, Ralph F. Lee ${ }^{1,2}$, Takahito Takezawa ${ }^{1}$, Keijiro Sunada ${ }^{1}$, Hironori Yamamoto ${ }^{1}$

1 Department of Medicine, Division of Gastroenterology, Jichi Medical University, Shimotsuke, Japan

2 Department of Medicine, Division of Gastroenterology, University of Ottawa, Ottawa, Ontario, Canada

\section{Corresponding author}

\section{Hironori Yamamoto, MD, PhD}

Department of Medicine, Division of Gastroenterology , Jichi Medical University, 3311-1 Yakushiji, Shimotsuke, Tochigi, 329-0498, Japan

Fax: +81-285-40-6598

ireef@jichi.ac.jp

\section{References}

[1] Sakamoto N, Osada T, Shibuya T et al. Endoscopic submucosal dissection of large colorectal tumors by using a novel spring-action S-O clip for traction (with video). Gastrointest Endosc 2009; 69: 1370-1374

[2] Nomura T, Kamei A, Sugimoto S et al. Colorectal endoscopic submucosal dissection using the "dental floss with rubber band method". Endoscopy 2018; 50: E78-E80

[3] Nomura T, Kamei A, Sugimoto $S$ et al. Colorectal endoscopic submucosal dissection using a clip-on-clip traction method. Endoscopy 2018; 50: E197-E198

[4] Hayashi Y, Sunada K, Takahashi H. Pocketcreation method of endoscopic submucosal dissection to achieve en bloc resection of giant colorectal subpedunculated neoplastic lesions. Endoscopy 2014; 46 (Suppl. 01): E421-E422

[5] Sakamoto H, Hayashi Y, Miura Y. Pocketcreation method facilitates endoscopic submucosal dissection of colorectal laterally spreading tumors, non-granular type. Endosc Int Open 2017; 5: E123-E129
Bibliography

DOI https://doi.org/10.1055/a-1066-4561

Published online: 20.12.2019

Endoscopy 2020; 52: E208-E210

(c) Georg Thieme Verlag KC

Stuttgart · New York

ISSN 0013-726X

\section{ENDOSCOPY E-VIDEOS}

https://eref.thieme.de/e-videos

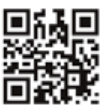

Endoscopy E-Videos is a free access online section, reporting on interesting cases and new

techniques in gastroenterological endoscopy. All papers include a high quality video and all contributions are freely accessible online.

This section has its own submission website at https://mc.manuscriptcentral.com/e-videos 